\title{
MRI-based radiomics models to assess prostate cancer, extracapsular extension and positive surgical margins
}

Dong $\mathrm{He}^{1 \dagger}$, Ximing Wang ${ }^{2 \dagger}$, Chenchao $\mathrm{Fu}^{1}$, Xuedong Wei ${ }^{1}$, Jie Bao ${ }^{2}$, Xuefu $\mathrm{Ji}^{3,4}$, Honglin Bai ${ }^{3}$, Wei Xia ${ }^{3}$, Xin Gao ${ }^{3}$, Yuhua Huang ${ }^{1 *}$ and Jianquan $\mathrm{Hou}^{1,5^{*}}$

\begin{abstract}
Purpose: To investigate the performance of magnetic resonance imaging (MRI)-based radiomics models for benign and malignant prostate lesion discrimination and extracapsular extension (ECE) and positive surgical margins (PSM) prediction.

Methods and materials: In total, 459 patients who underwent multiparametric MRI (mpMRI) before prostate biopsy were included. Radiomic features were extracted from both T2-weighted imaging (T2WI) and the apparent diffusion coefficient (ADC). Patients were divided into different training sets and testing sets for different targets according to a ratio of 7:3. Radiomics signatures were built using radiomic features on the training set, and integrated models were built by adding clinical characteristics. The areas under the receiver operating characteristic curves (AUCs) were calculated to assess the classification performance on the testing sets.

Results: The radiomics signatures for benign and malignant lesion discrimination achieved AUCs of 0.775 (T2WI), 0.863 (ADC) and 0.855 (ADC + T2WI). The corresponding integrated models improved the AUC to 0.851/0.912/0.905, respectively. The radiomics signatures for ECE achieved the highest AUC of 0.625 (ADC), and the corresponding integrated model achieved the highest AUC (0.728). The radiomics signatures for PSM prediction achieved AUCs of 0.614 (T2WI) and 0.733 (ADC). The corresponding integrated models reached AUCs of 0.680 and 0.766 , respectively.

Conclusions: The MRI-based radiomics models, which took advantage of radiomic features on ADC and T2WI scans, showed good performance in discriminating benign and malignant prostate lesions and predicting ECE and PSM. Combining radiomics signatures and clinical factors enhanced the performance of the models, which may contribute to clinical diagnosis and treatment.
\end{abstract}

Keywords: Prostate cancer, Radiomics, Extracapsular extension, Positive surgical margins

\footnotetext{
*Correspondence: sdfyy_hyh@163.com; xf192@163.com

${ }^{\dagger}$ Dong He and Ximing Wang contributed equally to this work.

'Department of Urology, The First Affiliated Hospital of SooChow University,

No.188, Shizi St, Canglang District, 215006 Suzhou, Jiangsu, China

Full list of author information is available at the end of the article
}

(C) The Author(s). 2021 Open Access This article is licensed under a Creative Commons Attribution 4.0 International License, which permits use, sharing, adaptation, distribution and reproduction in any medium or format, as long as you give appropriate credit to the original author(s) and the source, provide a link to the Creative Commons licence, and indicate if changes were made. The images or other third party material in this article are included in the article's Creative Commons licence, unless indicated otherwise in a credit line to the material. If material is not included in the article's Creative Commons licence and your intended use is not permitted by statutory regulation or exceeds the permitted use, you will need to obtain permission directly from the copyright holder. To view a copy of this licence, visit http://creativecommons.org/licenses/by/4.0/. The Creative Commons Public Domain Dedication waiver (http://creativecommons.org/publicdomain/zero/1.0/) applies to the data made available in this article, unless otherwise stated in a credit line to the data. 


\section{Introduction}

Prostate cancer $(\mathrm{PCa})$ is the second most common cancer in males worldwide [1]. According to the most recent cancer statistics estimated by the American Cancer Society, PCa alone accounted for nearly $20 \%$ of new cancer diagnoses and $10 \%$ of cancer deaths in males in 2019 [2].

The clinical gold standard for PCa diagnosis is prostate biopsy, but biopsy may lead to complications such as pain, bleeding, inflammation and dysuria [3-5]. Prostatespecific antigen (PSA) tests and digital rectal examinations (DREs) are widely used as non-invasive methods to detect PCa [6]. PSA tests and DREs have high sensitivity but low specificity [7].

After PCa is detected, staging is an important task that significantly influences management of the disease. The evaluation of extracapsular extension (ECE), which indicates that $\mathrm{PCa}$ has reached stage $\mathrm{T}_{3}$, is of significance because ECE is associated with cancer-specific survival and can affect the positive surgical margins (PSM) [8]. PSM is regarded as a negative prognostic factor in $\mathrm{PCa}$ patients [9]. The presence of PSM within a radical prostatectomy (RP) specimen has a negative effect on prognosis and is linked to a 3.7 -fold increase in the risk of biochemical recurrence [10].

To improve the risk assessment of ECE and PSM, many nomograms based on PSA, age, perineural invasion status, Gleason score and percentage of positive cores in biopsy pathology have been constructed [9, 11, 12]. However, ECE and PSM are still poorly predicted by standard clinical tests.

Multiparametric MRI (mpMRI) is considered a standard tool for diagnostic evaluations of $\mathrm{PCa}$ and can help reduce unnecessary biopsies by a quarter $[13,14]$. The accurate assessment of PCa by mpMRI before RP can help clinicians distinguish extraprostatic disease, identify risk factors associated with PSM, and evaluate intraoperative complications and functional recovery after surgery [15]. Several studies have demonstrated that the features extracted from T2WI and ADC can aid the classification of Gleason scores [16-19], indicating that the association between extraprostatic disease and the features extracted from ADC and T2WI can be used to improve the accuracy of benign and malignant prostate lesion discrimination and ECE and PSM prediction.

Radiomics is a novel tool that can translate images into meaningful data for analysis and has been applied in oncology and the development of machine learning methods [20]. Recent studies have explored the value of radiomics based on MRI in the differentiation of $\mathrm{PCa}$ from benign prostate tissue [21] and the evaluation of $\mathrm{PCa}$ aggressiveness [22]. Additional applications of radiomics are possible.
In this study, we attempted to investigate whether an MRI-based radiomics model could aid clinical diagnosis and treatment due to its efficiency in benign and malignant prostate lesion discrimination, ECE prediction and PSM prediction.

\section{Materials and methods}

\section{Patients and data collection}

With the approval of our institutional review board, we performed a retrospective analysis of 640 consecutive patients who underwent pelvic mpMRI and prostate biopsy at the First Affiliated Hospital of SooChow University.

Patients who met the following inclusion criteria were included in our study: (1) prostate lesions with welldefined boundaries on both T2WI and ADC images according to the Prostate Imaging Reporting and Data System version 2 (PI-RADS v2); (2) clinical information, including age, total PSA (tPSA), free PSA/tPSA (f/tPSA), biopsy Gleason score (biopsyGS), and percentage of positive cores; (3) mpMRI before biopsy; (4) trans-rectal ultrasound guided random biopsy including at least 12 cores; and (5) RP performed in PCa patients by the same urologist within three months after MRI and biopsy.

The exclusion criteria were as follows: (1) previous prostate biopsy; (2) previous treatment for $\mathrm{PCa}$; and (3) confirmed diagnosis of a tumor other than $\mathrm{PCa}$.

The following three biological characteristics were studied using the above patient data: discrimination of benign and malignant prostate lesions in all patients and ECE prediction and PSM prediction in patients with malignant prostate lesions.

\section{Magnetic resonance imaging protocols}

All patients were scanned with a 3.0T MRI scanner (MAGNETOM Skyra; Siemens Healthineers, Erlangen, Germany) using a standard spine array coil and an 18channel body array coil. The images included axial, coronal and sagittal T2WI (repetition time (TR)/echo time (TE), 3900/105 ms; flip angle, $160^{\circ}$; section thickness, 3 $\mathrm{mm}$; intersection gap, $0 \mathrm{~mm}$; FOV, $25 \mathrm{~cm}$; and matrix, 384*336) and axial DWI (diffusion weighted imaging) (b values, $0,700,1400$, and $2000 \mathrm{~s} / \mathrm{mm}^{2}$; TR/TE, 5000/72 ms; section thickness, $5 \mathrm{~mm}$; FOV, $20 \mathrm{~cm}$; and matrix, 128*128). ADC was obtained from DWI with b values of 0 and $700 \mathrm{~s} / \mathrm{mm}^{2}$ using a $2 \mathrm{D}$ echo planar imaging sequence.

\section{Pathological Evaluation}

After the biopsy or the prostatectomy, the excised tissue was submitted to histopathology. The RP specimen was fixed in formalin and sliced from apex to base in 3 to 4 $\mathrm{mm}$ intervals. These slices were then stained with haematoxylin and eosin (H\&E). A pathologist with 5 
years of experience outlined each lesion on the microscopic slices and assigned a Gleason score. ECE was determined by the presence of neoplastic tissue outside the prostatic capsular in the periprostatic tissue. PSM was determined by the presence of neoplastic tissue at the surgical margins. Another radiologist with over 5 years cooperated with the pathologist on the pathology-MRI lesion matching. All the results were confirmed and corrected by the other pathologist and radiologist both with over 15 years of experience.

\section{Radiomic feature extraction}

Experienced pathologists and radiologists reached a consensus regarding the standard histological-radiological correlation based on the histological and imaging findings. The tumor region of interest (ROI) was identified by anatomical landmarks and manually delineated slice by slice on both the T2WI and ADC sequences by radiologists using Medical Imaging Interaction Toolkit (MITK) software (version 2013.12.00) (Fig. 1). The full intensity range of the ROI was quantized to 32 Gy levels for subsequent feature extraction.

The radiomic features were extracted from the ROIs on both the T2WI and ADC sequences of each patient by using the open-source Python package Pyradiomics (version 2.1.0) [23]. The extracted features were divided into the following three categories: (1) 14 shape-based features, including descriptors of the three-dimensional size and shape of the ROI; (2) eighteen first-order features describing the distribution of the voxel intensities within the ROI using commonly used and basic metrics; and (3) 75 texture features, including the gray-level run-length matrix (GLRLM), gray-level co-occurrence matrix (GLCM), graylevel size-zone matrix (GLSZM), neighboring gray-tone difference matrix (NGTDM) and gray-level dependence matrix (GLDM). The wavelet features were calculated by wavelet decomposition to obtain the intensity and texture features of the original image. The features were concentrated in different frequency ranges within the tumor ROI [24]. In this research, all radiomic features, except for the shape-based features, were calculated based on the original image and derived image obtained by applying a wavelet transform. Ultimately, 851 quantitative radiomic features were extracted for each ROI.

\section{Feature selection}

The number of radiomic features was much larger than the number of patients, and feature selection was performed to avoid overfitting. A portion of the features may have low reproducibility when the ROI is manually delineated by different radiologists or at different times. To eliminate these features with low reproducibility, two radiologists (radiologist 1 with 6 years of experience in MRI interpretation and radiologist 2 with 10 years of experience) were assigned to delineate the ROIs in each case. Radiologist 1 performed a series of delineations at two different times, and radiologist 2 performed the delineation only once. The interclass and intraclass correlation coefficients (ICCs) were computed to assess the interobserver (radiologist 1 vs. radiologist 2) and intraobserver (radiologist 1) reliability, respectively. A large ICC value represents a high degree of reproducibility. Features with an ICC lower than 0.75 were considered to have poor agreement and were removed.

Features with a variance close to 0 were redundant and could not provide valid information for predicting the label because the values of such features barely change regardless of whether the case is negative or

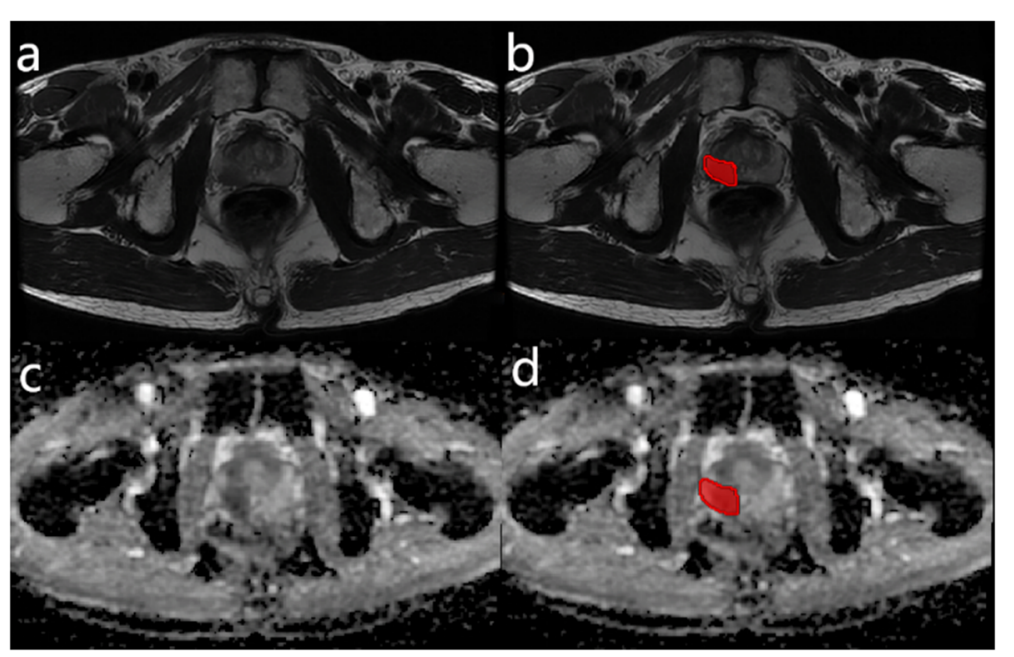

Fig. 1 Examples of ROI delineation. $\mathbf{a}$ and $\mathbf{c}$ are T2WI and ADC images, respectively, of the same patient. The red areas in $\mathbf{b}$ and $\mathbf{d}$ were delineated as ROls 
positive. A Spearman correlation analysis was performed to identify the highly correlated features [20]. Features with a mean absolute correlation higher than 0.9 were considered redundant. Standardization was performed to eliminate the impact of different feature orders of magnitude by scaling the features to a zero mean and unit variance.

Previous studies have shown that adding a prior feature ranking procedure may be helpful in improving the final performance. After eliminating the features with low reproducibility or high redundancy, we used featureranking algorithms to identify the most important features for label prediction based on a heuristic scoring criterion, and only the top-ranked features were retained. The minimum redundancy, maximum relevance (MRMR) approach is a representative and highly cited multivariate ranking method [25]. This method can find the $m$ features most relevant to the label from the feature space.

\section{Model building}

The least absolute shrinkage and selection operator (LASSO) regression algorithm was implemented to explore powerful predictive combinations of features related to labels and reduce overfitting and selection bias. We used the LASSO classifier to build radiomics signatures based on the top-ranking features of each single sequence and a multivariable logistic regression to build mpMRI signatures by combining predictions of different radiomics signatures. The classifier was trained using 10 -fold cross-validation and the training set.

To assess the impact of the clinical parameters, we built integrated models by adding the clinical characteristics to the radiomics signatures. We used independent $\mathrm{t}$-tests or Mann-Whitney $U$ tests to assess the continuous variables and chi-square tests to evaluate the categorical variables. The optimal model was chosen from combinations of radiomics signatures and subsets of clinical characteristics by using a multivariable logistic regression analysis and Akaike information criteria (AICs) [26].

The radiomics signatures and integrated models were tested using an independent testing set. The areas under the receiver operating characteristic (ROC) curves (AUCs) along with the $95 \%$ confidence intervals (CIs) and accuracy values were calculated to assess the classification performance, and the cut-off value was selected according to the Youden index to determine the corresponding sensitivity and specificity. The DeLong test [27] was used for the statistical comparison of the AUCs between the integrated models and the corresponding radiomics signatures.

The radiomics signature extracted from T2WI is denoted by $S_{T 2 W I}$, the signature extracted from ADC is denoted by $S_{A D C}$, and the signature of the combination of features extracted from T2WI and ADC is denoted by $S_{M P-M R I}$. The integrated diagnosis models are correspondingly denoted by $M_{T 2 W I}, M_{A D C}$ and $M_{M P-M R I}$.

$\mathrm{R}$ software (3.6.1) was used to conduct feature selection, model building, and statistical analysis.

\section{Results \\ Patient profiles}

In total, 459 patients with prostate disease (186 patients with benign tumors and 273 patients with malignant tumors) were analysed after screening according to the inclusion criteria (Fig. 2). 186 patients were diagnosed of benign diseases through biopsy and the other 273 patients with malignant tumors all underwent prostatectomy. The data included clinical information (age, tPSA, f/tPSA, positive core percentage, biopsyGS, MRI report and postoperative pathology report; Table 1) and pelvic mpMRI images. A stratified sampling method was used to divide the data into the training set and testing set at a ratio of 7:3. Of the 459 patients in this study, 323 were assigned to the training set, and 136 were assigned to the testing set. The ECE and PSM were studied using the 273 patients with malignant tumors, and the training and testing sets for these two labels contained 192 patients and 81 patients, respectively.

Between the $\mathrm{PCa}$ patient group and the non-PCa patient group, there were significant differences in age, tPSA and f/tPSA $(P<0.05)$. Both between the ECE patient group and the non-ECE patient group and between the PSM patient group and the non-PSM patient group, there were significant differences in tPSA, positive core percentage and biopsyGS. The details are shown in Tables S1, S2, S3.

There were no obvious differences in clinical characteristics between the different training sets and corresponding testing sets. The characteristics of the included patients are summarized in Tables S4, S5, S6; the PCa patients constituted 59.4 and $59.6 \%$ of the training and testing sets, respectively. The PCa patients with ECE constituted 41.7 and $40.7 \%$ of the training and testing sets, respectively. The patients with a PSM constituted $37.0 \%$ of both the training and testing sets. In the training and testing sets for benign and malignant prostate lesion discrimination, there were no significant differences in age, tPSA, or $\mathrm{f} / \mathrm{tPSA}(P>0.05$; Table S7), and in the training and testing sets used for ECE and PSM prediction, there were no significant differences in tPSA, positive core percentage and biopsyGS $(P>0.05$; Tables S8 and S9).

Feature selection and acquisition of radiomics signatures In total, 851 radiomic features per patient were extracted separately from the ROIs on the T2WI and ADC images. After removing features with low reproducibility or high redundancy, 278 T2WI features and 281 ADC features 


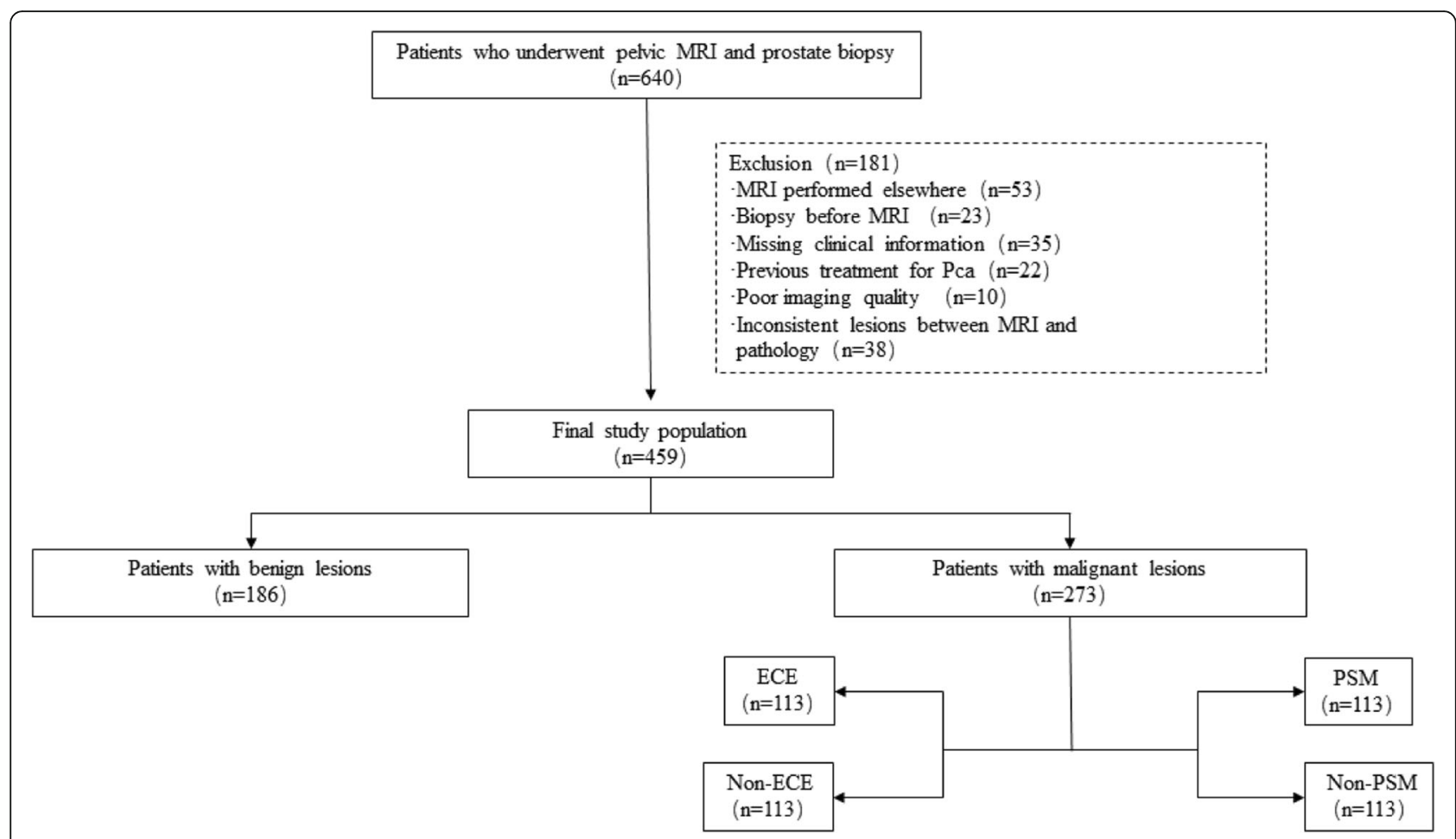

Fig. 2 Flow diagram of patient selection for the study

were retained for benign and malignant prostate lesion discrimination, 278 T2WI features and 293 ADC features were retained for ECE prediction, and 268 T2WI features and 295 ADC features were retained for PSM prediction. The remaining features were ranked by the MRMR approach, and the top 10 features were selected as the optimal radiomic feature subset.

The LASSO classifier was trained using the training set and the optimal radiomic feature subset to build the

Table 1 Clinical information of patients with benign and malignant prostate disease

\begin{tabular}{|c|c|c|}
\hline & Benign & Malignant \\
\hline Number & 186 & 273 \\
\hline Age, mean (range) (year) & $65.23(30-89)$ & $70.21(49-87)$ \\
\hline tPSA $(n g / m l)$, mean (range) & $13.62(1.64-136.45)$ & $28.95(2.85-211.87)$ \\
\hline f/tPSA, mean (range) & $0.16(0.03-0.40)$ & $0.13(0.02-0.43)$ \\
\hline PI-RADS V2, median (range) & $3(2-5)$ & $4(3-5)$ \\
\hline Positive core percentage, mean (range) & - & $0.41(0.08-1)$ \\
\hline BiopsyGS, median (range) & - & $7(6-10)$ \\
\hline ECE reported by MRI & - & 25 \\
\hline ECE on postoperative pathology, $n$ & - & 113 \\
\hline reported by MRI, n (\%) & - & $23(20.4)$ \\
\hline not reported by MRI, n (\%) & - & $90(79.6)$ \\
\hline PSM on postoperative pathology, $n$ & - & 101 \\
\hline PSM with ECE, n (\%) & - & $68(67.3)$ \\
\hline PSM without ECE, n (\%) & - & $33(32.7)$ \\
\hline Pathologic stage & - & \\
\hline pT2, n (\%) & - & $160(58.6)$ \\
\hline pT3, n (\%) & - & $113(41.4)$ \\
\hline
\end{tabular}


radiomics signatures. The details of the features used to build the different signatures are shown in Tables S10, S11, S12. These tables also show the positive and negative correlations and dependencies between the features and labels by providing the $p$-values and Spearman's $r$ coefficients.

After the multivariable logistic regression analysis and AIC analysis, different combinations of $S_{T 2 W I}, S_{A D C}$, age, tPSA, f/tPSA, biopsyGS and positive core percentage were selected to build integrated models. The details and formulas are shown in Tables S13, S14, S15, S16, S17, S18, S19, S20, S21, S22, S23, S24, S25, S26, S27.

The performances of the radiomics signatures and integrated models of different characteristics are shown in Tables 2, 3 and 4. $M_{A D C}$ yielded the highest AUC of 0.912 for benign and malignant prostate lesion discrimination. $M_{A D C}$ achieved the highest AUC of 0.728 for ECE prediction. $M_{A D C}$ yielded the highest AUC of 0.766 for PSM prediction. The $p$-values of the DeLong tests, $95 \% \mathrm{CIs}$ and the accuracy, sensitivity and specificity values are also shown in Tables 2, 3 and 4.

The ROC curves of the different radiomics signatures, the integrated models and the combination of the radiomics signatures and integrated models for evaluating the effects of adding clinical information are shown in Fig. 3.

\section{Discussion}

Despite the reliability of senior radiologists in detecting cancer lesions and boundaries, it is still challenging to determine the aggressiveness of $\mathrm{PCa}$ and predict the effect of surgical treatment. When estimating potential lesions, clinicians are limited to their personal knowledge and previous experience. These estimates are influenced by subjective judgements that may lead to variability among different clinicians. Machine learning based on the radiomics method can extract a large number of distinctive imaging features beyond those obtained by visual analysis by clinicians and build an objective

Table 2 The performance of radiomics signatures in the testing set for benign and malignant prostate lesion discrimination

\begin{tabular}{lcclllll}
\hline & AUC & $\boldsymbol{p}$-value & $\mathbf{9 5} \% \mathbf{C l}$ & ACC & SEN & SPE & Cut-off \\
\hline$S_{T 2 W I}$ & 0.775 & 0.008 & $0.696,0.842$ & 0.699 & 0.654 & 0.782 & 0.622 \\
$M_{T 2 W I}$ & 0.851 & & $0.780,0.907$ & 0.794 & 0.840 & 0.727 & 0.463 \\
$S_{A D C}$ & 0.863 & 0.024 & $0.793,0.916$ & 0.809 & 0.827 & 0.781 & 0.620 \\
$M_{A D C}$ & 0.912 & & $0.852,0.954$ & 0.868 & 0.877 & 0.873 & 0.552 \\
$S_{M P-M R I}$ & 0.855 & 0.010 & $0.784,0.909$ & 0.801 & 0.815 & 0.782 & 0.574 \\
$M_{M P-M R I}$ & 0.905 & & $0.843,0.949$ & 0.846 & 0.790 & 0.927 & 0.678
\end{tabular}

The $p$-values were derived from DeLong tests. The first row of $p$-values compares the AUCs of the radiomics signatures with random guesses by chance (AUC of 0.5), and the second row compares the AUCs of the radiomics signatures with those of the corresponding integrated models
Table 3 The performance of radiomics signatures in the testing set for ECE prediction

\begin{tabular}{lllllllc}
\hline & AUC & $\boldsymbol{p}$-value & $\mathbf{9 5} \% \mathbf{C l}$ & ACC & SEN & SPE & Cut-off \\
\hline$S_{\text {T2WI }}$ & 0.599 & 0.002 & $0.484,0.707$ & 0.617 & 0.636 & 0.625 & 0.396 \\
$M_{T 2 W I}$ & 0.726 & & $0.616,0.819$ & 0.691 & 0.849 & 0.583 & 0.267 \\
$S_{A D C}$ & 0.625 & 0.007 & $0.500,0.724$ & 0.580 & 0.697 & 0.521 & 0.386 \\
$M_{A D C}$ & 0.728 & & $0.618,0.821$ & 0.691 & 0.727 & 0.688 & 0.427 \\
\hline
\end{tabular}

prediction model. In our work, T2WI and ADC were used to build radiomics signatures for benign and malignant prostate lesion discrimination, ECE prediction and PSM prediction.

ADC and T2WI have been suggested to be negatively correlated with the percentage area of nuclei or cytoplasm measured in histopathological prostate tissue specimens and positively correlated with the percentage of luminal space [28]. With higher Gleason scores, a disrupted gland architecture results in a more chaotic and scattered appearance of the gland lumen [29]. The above findings were considered the histological basis of the correlations between PCa and the features on ADC and T2WI. In addition, $\mathrm{ADC}$ has been proven to provide more information than T2WI. In addition to providing information on cellularity, cell count and epithelial volume, ADC is inversely correlated with the expression of $\mathrm{Ki}-67$, which is associated with proliferation potential [30].

The radiomic features ranked by the MRMR approach and then used in the radiomics signatures by LASSO were mostly wavelet-based features $(8 / 10$ for tumor discrimination, $3 / 6$ for ECE prediction and $3 / 4$ for PSM prediction). Wavelet-based features have a strong ability to predict labels and can quantify the heterogeneity of tumors at different scales that cannot be recognized by the naked eye [31]. These features play an important role in the establishment of radiomics signatures.

The radiomics signatures performed well in benign and malignant prostate lesion discrimination with the highest AUCs of 0.863 (ADC) and 0.775 (T2WI). The integrated model using the combination of the ADC signatures and clinical information reached an AUC of 0.912 and thus was better than the model combining the mpMRI signatures and clinical information. These findings suggest that the ADC sequence had more valid information for benign and malignant prostate lesion

Table 4 The performance of radiomics signatures in the testing set for PSM prediction

\begin{tabular}{lccccccc}
\hline & AUC & $\boldsymbol{p}$-value & $\mathbf{9 5} \% \mathbf{C l}$ & ACC & SEN & SPE & Cut-off \\
\hline$S_{T 2 W I}$ & 0.614 & 0.099 & $0.499,0.720$ & 0.605 & 0.800 & 0.431 & 0.368 \\
$M_{T 2 W I}$ & 0.680 & & $0.567,0.779$ & 0.691 & 0.633 & 0.745 & 0.414 \\
$S_{A D C}$ & 0.733 & 0.244 & $0.623,0.826$ & 0.667 & 0.867 & 0.569 & 0.311 \\
$M_{A D C}$ & 0.766 & & $0.659,0.853$ & 0.728 & 0.667 & 0.765 & 0.360 \\
\hline
\end{tabular}



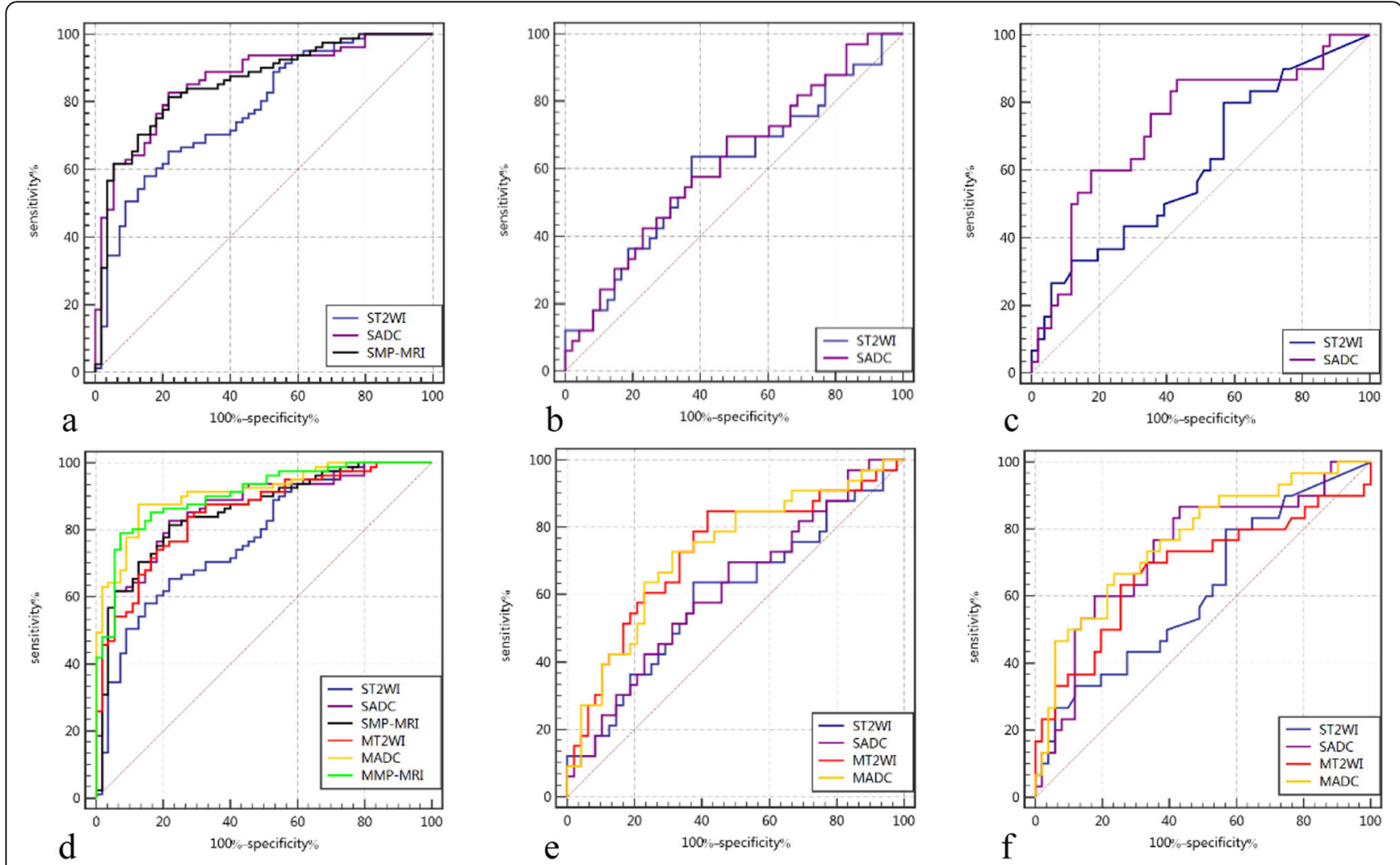

Fig. 3 ROC curves of the radiomics signatures. (a) Radiomics signatures for benign and malignant prostate lesion discrimination. (b) Radiomics signatures for ECE prediction. (c) Radiomics signatures for PSM prediction. (d), (e), (f). Combinations of the radiomics signatures and the integrated models for the three tasks separately to evaluate the effect of adding clinical information

discrimination than the T2WI sequence. According to Table S4, age and f/tPSA were both included in the three integrated models. The clinical information and radiomic features were complementary and could be combined to obtain the prostate-related characteristics. Recent studies based on radiomics have used different features from different MRI sequences to discriminate benign prostate lesions from malignant lesions with AUCs ranging from 0.70 to 0.92 [21, 32]. Our findings are consistent with these studies, and we incorporated more clinical data from the patients for model optimization.

The radiomics signatures for ECE prediction achieved the highest AUC of 0.625 using the ADC sequence. Notably, among the 113 patients with ECE diagnosed by postoperative pathology, only 23 patients $(20.3 \%)$ were identified correctly by the preoperative MRI report with the manual identification method. There should be a correlation within in the radiomic features extracted from primary localized malignant lesions between the occurrence of ECE and tumor heterogeneity. The integrated models improved the performance to an AUC of 0.728 compared with the corresponding radiomics signatures with no clinical information. These results suggest that it is difficult to predict ECE using only preoperative
MRI and that the inclusion of clinical information is necessary.

Previously, the factors used to predict PSM after RP in PCa mainly relied on clinical information. Turan et al. [33] identified that a positive core percentage of biopsy specimens, tPSA levels and the elapsed time between biopsy and surgery increased the risk of a PSM. Yang et al. [11] reported that perineural invasion, higher biopsy Gleason scores and more positive cores in biopsy specimens were independent predictors of a PSM. Although other predictors have been reported, such as body mass index, tPSA, prostate volume, and surgical experience, previous data have been largely inconclusive [34]. Considering the increasingly important role of mpMRI in the evaluation of $\mathrm{PCa}$, there could be a potential association between radiomics and the occurrence of PSM. In this study, we selected patients whose operations were performed by the same senior urologist. As a result, the radiomics signatures performed well in the PSM prediction, with the highest AUC of 0.733 using ADC, and the corresponding intergraded model reached an AUC of 0.766 . Radiomics signatures might reflect a number of characteristics. First, aggressive cancerous tissue blurs the boundary between normal and cancerous tissue under surgical vision, making it difficult to determine 
the boundary during surgery. Second, malignant tumor tissue loses stable tissue structure, increasing its susceptibility to invasion by surgical instruments compared with normal tissue during surgery.

Early studies have confirmed that age, tPSA and f/ tPSA are significant parameters in PCa diagnosis [7]. The percentage of positive cores and the Gleason score based on biopsy pathology have been proven to be correlated with the aggressiveness of $\mathrm{PCa}[11,35]$. In our study, clinical data were fully utilized. A multivariable logistic regression analysis was conducted, and the AICs were compared to select risk factors from the above parameters for inclusion in different integrated models for optimization.

Prior studies have used machine learning models analyzing radiomic features to detect $\mathrm{PCa}$ and predict Gleason scores $[16,18,21]$. Some studies have demonstrated good correlations of mpMRI with other variables and ECE [36-38]. Alves et al. [39] achieved good results in an independent external validation of a nomogram using clinical data and imaging to predict ECE. Our study comprehensively analysed clinical variables and imaging features, and the results were consistent with previous studies. In addition, our experiments involved a relatively large amount of patient data, and we filtered potentially useful information from a larger number of possible features. Moreover, we explored the implied information contained in radiomic features in the prediction of ECE and PSM. We believe that applying our model could provide a reference for clinical judgment, thereby potentially improving diagnostic, prognostic, and predictive accuracy.

Our work has several limitations. First, the analysis was retrospectively performed using a dataset obtained at a single center. Further verification should be confirmed in a multi-center clinical study to estimate practicability. Second, we did not reanalyze the postoperative pathological slices but extracted information from pathology reports. Some uncertainty might exist in the match between the ROIs used to extract the radiomic features and the corresponding ROIs defined in the histological slices. Third, while DWI and dynamic contrastenhanced MRI (DCE-MRI) have been proven to be accurate assessments of $\mathrm{PCa}$, these methods were not included in our study because ADC is calculated from DWI; thus, there is an intrinsic connection between them, and the importance of DCE-MRI has diminished [40]. Furthermore, we did not apply radiomics assessment to lesions in different areas, such as peripheral and transitional zones and areas that were not well-defined.

In conclusion, our study demonstrates that radiomics signatures based on MRI could be used as a predictor in the discrimination of benign and malignant prostate lesions and the prediction of ECE and PSM. Prediction models were established, and we found that the combination of clinical data and radiomics signatures could improve the efficiency of the model compared to models including only radiomic features. Our findings might help clinicians better identify the risk of PCa compared with previous methods. Furthermore, wider tissue resection and more careful operation in PCa surgery should be considered when our model predicts risks of ECE and PSM.

\section{Abbreviations \\ ADC: Apparent diffusion coefficient; ECE: Extracapsular extension; LASSO: Least absolute shrinkage and selection operator; MP-MRI: Multiparametric MRI; MRMR: Minimum redundancy maximum relevance; PCa: Prostate cancer; ROI: Region of interest; T2WI: T2-weighted imaging}

\section{Supplementary Information}

The online version contains supplementary material available at https://doi. org/10.1186/s40644-021-00414-6.

\section{Additional file 1:}

\section{Acknowledgements}

The authors would like to thank the Departments of Urology, Radiology, and Pathology of the First Affiliated Hospital of SooChow University and Suzhou Institute of Biomedical Engineering and Technology, Chinese Academy of Sciences for their valuable help.

\section{Authors' contributions \\ Guarantor of integrity of the entire study: $\mathrm{HYH}, \mathrm{HJQ}$; study concepts and design: WXD, GX, XW; literature research: XW, WXD, WXM ; data collection: FCC, BJ; HD; data analysis: BHL, JXF; manuscript preparation: HD, WXM; manuscript review: HYH, XW, WXD. All authors read and approved the final manuscript.}

\section{Funding}

This study has received funding by Suzhou science and technology plan project [grant numbers SS201863 and SYG201908], Suzhou Science and Technology Bureau-Science and Technology Demonstration Project [grant number SS201808], and National Natural Science Foundation of China [grant number 61801474].

\section{Availability of data and materials}

The datasets used and/or analysed during the current study are available from the corresponding author on reasonable request.

\section{Declarations}

Ethics approval and consent to participate

Institutional Review Board approval was obtained in concordance with the standards of The First Affiliated Hospital of Soochow University Ethics

Committee (2020; approval no. 064).

Consent for publication

Not applicable.

\section{Competing interests}

The authors declare that they have no competing interests.

\section{Author details}

${ }^{1}$ Department of Urology, The First Affiliated Hospital of SooChow University, No.188, Shizi St, Canglang District, 215006 Suzhou, Jiangsu, China.

${ }^{2}$ Department of Radiology, The First Affiliated Hospital of SooChow University, No.188, Shizi St, Canglang District, 215006 Suzhou, Jiangsu, China. ${ }^{3}$ Suzhou Institute of Biomedical Engineering and Technology, Chinese Academy of Sciences, No.88 Keling Road, Suzhou New District, 215163 Jiangsu, China. ${ }^{4}$ The School of Electro-Optical Engineering, Changchun 
University of Science and Technology, 130013 Changchun, China. ${ }^{5}$ Department of Urology, Dushu Lake Hospital affiliated to SooChow University, No.9, Chongwen Road, Suzhou Industrial Park District, Suzhou, Jiangsu 215000, China.

Received: 25 February 2021 Accepted: 10 June 2021

Published online: 05 July 2021

\section{References}

1. Hernandez G, Ramirez JL, Pedroza-Torres A, Herrera LA, Jimenez-Rios MA. The secret life of translation initiation in prostate cancer. Front Genet. 2019;10:14.

2. Siegel RL, Miller KD, Jemal A. Cancer statistics, 2019. CA Cancer J Clin. 2019; 69:7-34.

3. Moore CM, Robertson NL, Arsanious N, et al. Image-guided prostate biopsy using magnetic resonance imaging-derived targets: a systematic review. Eur Urol. 2013;63:125-40

4. Del Monte M, Leonardo C, Salvo V, et al. MRI/US fusion-guided biopsy: performing exclusively targeted biopsies for the early detection of prostate cancer. Radiol Med. 2018:123:227-34.

5. Rosario DJ, Lane JA, Metcalfe C, et al. Short term outcomes of prostate biopsy in men tested for cancer by prostate specific antigen: prospective evaluation within ProtecT study. Bmj. 2012;344:d7894.

6. Barry MJ, Simmons LH. Prevention of Prostate Cancer Morbidity and Mortality: Primary Prevention and Early Detection. Med Clin North Am. 2017; 101:787-806.

7. Schroder FH, Hugosson J, Roobol MJ, et al. Screening and prostate-cancer mortality in a randomized European study. N Engl J Med. 2009:360:1320-8.

8. Tollefson MK, Karnes RJ, Rangel $\sqcup$, Bergstralh EJ, Boorjian SA. The impact of clinical stage on prostate cancer survival following radical prostatectomy. J Urol. 2013;189:1707-12

9. Zhang $L$, Wu B, Zha Z, et al. Surgical margin status and its impact on prostate cancer prognosis after radical prostatectomy: a meta-analysis. World J Urol. 2018:36:1803-15.

10. Karakiewicz PI, Eastham JA, Graefen M, et al. Prognostic impact of positive surgical margins in surgically treated prostate cancer: multi-institutional assessment of 5831 patients. Urology. 2005:66:1245-50.

11. Yang R, Cao K, Han T, et al. Perineural invasion status, Gleason score and number of positive cores in biopsy pathology are predictors of positive surgical margin following laparoscopic radical prostatectomy. Asian J Androl. 2017;19:468-72.

12. Ohori M, Kattan MW, Koh $\mathrm{H}$, et al. Predicting the presence and side of extracapsular extension: a nomogram for staging prostate cancer. J Urol. 2004;171:1844-9. discussion 1849.

13. Patel $P$, Wang $S$, Siddiqui MM. The use of multiparametric magnetic resonance imaging ( $\mathrm{mpMRI}$ ) in the detection, evaluation, and surveillance of clinically significant prostate cancer (csPCa). Curr Urol Rep. 2019;20:60.

14. Álvarez-Maestro M, Gómez Rivas J, Quesada Olarte J, et al. Magnetic resonance as imaging diagnostic tool in prostate cancer: New evidences-The EAU Section of Uro-Technology position. Actas Urol Esp. 2020;44:148-55.

15. McEvoy SH, Raeside MC, Chaim J, Ehdaie B, Akin O. Preoperative Prostate MRI: A Road Map for Surgery. AJR Am J Roentgenol. 2018;211:383-91.

16. Fehr $D$, Veeraraghavan $H$, Wibmer $A$, et al. Automatic classification of prostate cancer Gleason scores from multiparametric magnetic resonance images. Proc Natl Acad Sci U S A. 2015;112:E6265-73.

17. Nketiah G, Elschot M, Kim E, et al. T2-weighted MRI-derived textural features reflect prostate cancer aggressiveness: preliminary results. Eur Radiol. 2017; 27:3050-9.

18. Penzias $G$, Singanamalli $A$, Elliott $R$, et al. Identifying the morphologic basis for radiomic features in distinguishing different Gleason grades of prostate cancer on MRI: Preliminary findings. PLoS One. 2018;13:e0200730.

19. Chatterjee A, Watson G, Myint E, Sved P, McEntee M, Bourne R. Changes in Epithelium, Stroma, and Lumen Space Correlate More Strongly with Gleason Pattern and Are Stronger Predictors of Prostate ADC Changes than Cellularity Metrics. Radiology. 2015;277:751-62.

20. Gillies RJ, Kinahan PE, Hricak H. Radiomics: images are more than pictures, they are data. Radiology. 2016;278:563-77.

21. Xu M, Fang M, Zou J, et al. Using biparametric MRI radiomics signature to differentiate between benign and malignant prostate lesions. Eur J Radiol. 2019;114:38-44.

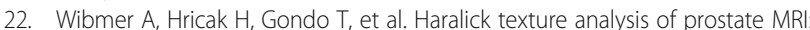
utility for differentiating non-cancerous prostate from prostate cancer and differentiating prostate cancers with different Gleason scores. Eur Radiol. 2015;25:2840-50.

23. van Griethuysen JJM, Fedorov A, Parmar C, et al. Computational radiomics system to decode the radiographic phenotype. Cancer Res. 2017;77:e104-7.

24. Aerts HJ, Velazquez ER, Leijenaar RT, et al. Decoding tumour phenotype by noninvasive imaging using a quantitative radiomics approach. Nat Commun. 2014;5:4006

25. Peng $H$, Long $F$, Ding $C$. Feature selection based on mutual information: criteria of max-dependency, max-relevance, and min-redundancy. IEEE Trans Pattern Anal Mach Intell. 2005;27:1226-38.

26. Huang YQ, Liang $\mathrm{CH}$, He $\mathrm{L}$, et al. Development and validation of a radiomics nomogram for preoperative prediction of lymph node metastasis in colorectal cancer. J Clin Oncol. 2016;34:2157-64.

27. DeLong ER, DeLong DM, Clarke-Pearson DL. Comparing the areas under two or more correlated receiver operating characteristic curves: a nonparametric approach. Biometrics. 1988;44:837-45.

28. Langer $\mathrm{DL}$, van der Kwast $\mathrm{TH}$, Evans AJ, et al. Prostate tissue composition and MR measurements: investigating the relationships between ADC, T2, K(trans), v(e), and corresponding histologic features. Radiology. 2010;255: 485-94.

29. Epstein Jl. An update of the Gleason grading system. J Urol. 2010;183:433-40.

30. Surov A, Meyer HJ, Wienke A. Correlations between apparent diffusion coefficient and gleason score in prostate cancer: a systematic review. Eur Urol Oncol. 2019;3:489-97.

31. Meng X, Xia W, Xie $P$, et al. Preoperative radiomic signature based on multiparametric magnetic resonance imaging for noninvasive evaluation of biological characteristics in rectal cancer. Eur Radiol. 2019;29:3200-9.

32. Litjens GJ, Elliott R, Shih NN, et al. Computer-extracted Features Can Distinguish Noncancerous Confounding Disease from Prostatic Adenocarcinoma at Multiparametric MR Imaging. Radiology. 2016;278:135-45.

33. Turan T, Boylu U, Basatac C, Gumus E. Predictive preoperative factors for positive surgical margins in robotic radical prostatectomy in low-risk prostate cancer. Turk J Urol. 2013;39:69-73.

34. Yossepowitch O, Briganti A, Eastham JA, et al. Positive surgical margins after radical prostatectomy: a systematic review and contemporary update. Eur Urol. 2014;65:303-13.

35. Tarjan M, Tot T. Prediction of extracapsular extension of prostate cancer based on systematic core biopsies. Scand J Urol Nephrol. 2006;40:459-64.

36. Rosenkrantz AB, Chandarana $\mathrm{H}$, Gilet $\mathrm{A}$, et al. Prostate cancer: utility of diffusion-weighted imaging as a marker of side-specific risk of extracapsular extension. J Magn Reson Imaging. 2013;38:312-9.

37. Sertdemir M, Weidner AM, Schoenberg SO, et al. Is There a Role for Functional MRI for the Assessment of Extracapsular Extension in Prostate Cancer? Anticancer Res. 2018;38:427-32.

38. Woo S, Cho JY, Kim SY, Kim SH. Extracapsular extension in prostate cancer: added value of diffusion-weighted MRI in patients with equivocal findings on T2-weighted imaging. AJR Am J Roentgenol. 2015;204:W168-75.

39. Alves JR, Muglia VF, Lucchesi FR, et al. Independent external validation of nomogram to predict extracapsular extension in patients with prostate cancer. Eur Radiol. 2020;30:5004-10.

40. Steiger P, Thoeny HC. Prostate MRI based on PI-RADS version 2: how we review and report. Cancer Imaging. 2016;16:9.

\section{Publisher's Note}

Springer Nature remains neutral with regard to jurisdictional claims in published maps and institutional affiliations.

Ready to submit your research? Choose BMC and benefit from:

- fast, convenient online submission

- thorough peer review by experienced researchers in your field

- rapid publication on acceptance

- support for research data, including large and complex data types

- gold Open Access which fosters wider collaboration and increased citations

- maximum visibility for your research: over $100 \mathrm{M}$ website views per year

At $\mathrm{BMC}$, research is always in progress.

Learn more biomedcentral.com/submission 